\title{
EXISTENCE OF EIGENVALUES OF A LINEAR OPERATOR PENCIL IN A CURVED WAVEGUIDE-LOCALIZED SHELF WAVES ON A CURVED COAST*
}

\author{
E. R. JOHNSON ${ }^{\dagger}$, MICHAEL LEVITIN $^{\ddagger}$, AND LEONID PARNOVSKI ${ }^{\dagger}$
}

\begin{abstract}
The question of the existence of nonpropagating, trapped continental shelf waves (CSWs) along curved coasts reduces mathematically to a spectral problem for a self-adjoint operator pencil in a curved strip. Using methods developed for the waveguide trapped mode problem, we show that such CSWs exist for a wide class of coast curvature and depth profiles.
\end{abstract} pencil

Key words. continental shelf waves, curved coasts, trapped modes, essential spectrum, operator

AMS subject classifications. 35P05, 35P15, 86A05, 76U05

DOI. $10.1137 / 040615936$

1. Introduction. Measurements of velocity fields along the coasts of oceans throughout the world show that much of the fluid energy is contained in motions with periods of a few days or longer. The comparison of measurements at different places along the same coast shows that in general these low-frequency disturbances propagate along coasts with shallow water to the right in the northern hemisphere and to the left in the southern hemisphere. These waves have come to be known as continental shelf waves (CSWs). The purpose of the present paper is to demonstrate, using the most straightforward model possible, the possibility of nonpropagating, trapped CSWs along curved coasts. The existence of such nonpropagating modes would be significant as they would tend to be forced by atmospheric weather systems, which have similar periods of a few days, similar horizontal extent, and a reasonably broad spectrum in space and time. Areas where such modes were trapped would thus appear to be likely to show higher than normal energy in the low-frequency horizontal velocity field.

The simplest models for CSWs take the coastal oceans to be inviscid and of constant density. Both these assumptions might be expected to fail in various regions such as when strong currents pass sharp capes or when the coastal flow is strongly stratified. However, for small-amplitude CSWs in quiescent flow along smooth coasts, viscous separation is negligible. Similarly most disturbance energy is concentrated in the modes with the least vertical structure, which are well described by the constant density model [LBMy]. The governing equations are then simply the rotating incompressible Euler equations. Further, coastal flows are shallow in the sense that the ratio of depth to typical horizontal scale is small. Expanding the rotating incompressible Euler equations in powers of this ratio and retaining only the leading order terms

*Received by the editors September 29, 2004; accepted for publication (in revised form) June 7, 2005; published electronically January 12, 2006.

http://www.siam.org/journals/sima/37-5/61593.html

${ }^{\dagger}$ Department of Mathematics, University College London, Gower Street, London WC1E 6BT, UK (e.johnson@ucl.ac.uk, leonid@math.ucl.ac.uk). The research of the third author was partially supported by the EPSRC Spectral Theory Network.

${ }^{\ddagger}$ Maxwell Institute for Mathematical Sciences, Department of Mathematics, Heriot-Watt University, Riccarton, Edinburgh EH14 4AS, UK (m.levitin@ma.hw.ac.uk). The research of this author was partially supported by the EPSRC Spectral Theory Network. 
gives the rotating shallow water equations $[\mathrm{Pe}]$ :

$$
\begin{aligned}
\frac{\partial \mathbf{u}}{\partial t}+\mathbf{u} \cdot \operatorname{grad} \mathbf{u}-2 \Omega \mathbf{k} \times \mathbf{u} & =-g \operatorname{grad} \widetilde{H} \\
\frac{\partial \widetilde{H}}{\partial t}+\operatorname{div}[(\widetilde{H}+H) \mathbf{u}] & =0 .
\end{aligned}
$$

Here div and grad are taken with respect to horizontal coordinates $(x, y)$ in a frame fixed to the rotating Earth, $\mathbf{k}$ is a vertical unit vector, $\mathbf{u}(x, y, t)$ is the horizontal velocity (with components $\mathbf{u}=(u, v)), \Omega$ is the (locally constant) vertical component of the Earth's rotation, $g$ is the gravitational acceleration, $\widetilde{H}(x, y, t)$ is the vertical displacement of the free surface, and $H(x, y)$ is the local undisturbed fluid depth.

System (1.1), (1.2) admits waves of two types, denoted Class 1 and Class 2 by [La]. Class 1 waves are fast high-frequency waves, the rotation-modified form of the usual free surface water waves, although here present only as long, nondispersive waves with speeds of order $\sqrt{g H}$. Class 2 waves are slower, low-frequency waves that vanish in the absence of depth change or in the absence of rotation. It is the Class 2 waves that give CSWs. They have little signature in the vertical height field $\widetilde{H}(x, y, t)$ and are observed through their associated horizontal velocity fields [Ha]. The Class 1 waves can be removed from (1.1), (1.2) by considering the "rigid-lid" limit, where the external Rossby radius $\sqrt{g H} / 2 \Omega$ (which gives the relaxation distance of the free surface) is large compared to the horizontal scale of the motion. This is perhaps the most accurate of the approximations noted here, causing the time-dependent term to vanish from (1.2) and the right side of (1.1) to become a simple pressure gradient.

For small amplitude waves the nonlinear terms in (1.1), (1.2) are negligible, and cross-differentiating gives

$$
\begin{aligned}
\frac{\partial \zeta}{\partial t}+2 \Omega \operatorname{div} \mathbf{u} & =0, \\
\operatorname{div}(H \mathbf{u}) & =0,
\end{aligned}
$$

where $\zeta=\frac{\partial v}{\partial x}-\frac{\partial u}{\partial y}$ is the vertical component of relative vorticity. Equation (1.4) is satisfied by introducing the volume flux streamfunction defined through

$$
H u=-\frac{\partial \psi}{\partial y}, \quad H v=\frac{\partial \psi}{\partial x},
$$

allowing (1.3) to be written as the single equation

$$
\operatorname{div}\left(\frac{1}{H} \operatorname{grad} \frac{\partial \psi}{\partial t}\right)+2 \Omega \mathbf{k} \cdot \operatorname{grad} \psi \times \operatorname{grad}\left(\frac{1}{H}\right)=0 .
$$

Equation (1.6) is generally described as the topographic Rossby wave equation or the equation for barotropic CSWs. Many solutions have been presented for straight coasts, where the coast lies along $y=0$ (say) and the depth $H$ is a function of $y$ alone (described as rectilinear topography here) [LBMy]. These have shown excellent agreement with observations of CSWs, as in [Ha]. There has been far less discussion of nonrectilinear geometries, where either the coast or the depth profile or both are not functions of a single coordinate. Yet interesting results appear. The papers [StHu1], [StHu2] present extensive numerical integrations of a low-order spectral model of a rectangular lake with idealized topography. For their chosen depth 
profiles normal modes can be divided into two types: basin-wide modes which extend throughout the lake and localized bay modes. These bay modes correspond to the high-frequency modes found in a finite-element model of Lake Lugarno by [Tr] and observed by [StHuSaTrZa]. The papers [Jo2], [StJo1], [StJo2] give a variational formulation and describe simplified quasi-analytical models that admit localized trapped bay modes. However the geometry changes in these models are large, with the sloping lower boundary terminating abruptly where it strikes a coastal wall. Further [Jo1] notes that (1.6) is invariant under conformal mappings and so any geometry that can be mapped conformally to a rectilinear shelf cannot support trapped modes. The question thus arises as to whether it is only for the most extreme topographic changes that shelf waves can be trapped or whether trapping can occur on smoothly varying shelves. The purpose of this paper is to provide the answer: trapped modes can exist on smoothly curving coasts.

The geometry considered here is that of a shelf of finite width lying along an impermeable coast. Thus sufficiently far from the coast the undisturbed fluid depth becomes the constant depth of the open ocean. It is shown in [Jo3] that at the shelfocean boundary of finite-width rectilinear shelves the tangential velocity component $u$ vanishes for waves sufficiently long compared to the shelf width. The wavelength of long propagating disturbances is proportional to their frequency which is in turn proportional to the slope of the shelf. Thus it appears that for sufficiently weakly sloping shelves the tangential velocity component, i.e., the normal derivative of the streamfunction, at the shelf-ocean boundary can be made arbitrarily small. Here this will be taken as also giving a close approximation to the boundary condition at the shelf-ocean boundary when this boundary is no longer straight. The unapproximated boundary condition is that the streamfunction and its normal derivative are continuous across the boundary where they match to the decaying solution of Laplace's equation (to which (1.6) reduces in regions of constant depth). This gives a linear integral condition along the boundary. The unapproximated problem will not be pursued further here. The boundary condition at the coast is simply one of impermeability and thus on both rectilinear and curving coasts is simply that the streamfunction vanishes. Now consider flows of the form

$$
\psi(x, y, t)=\operatorname{Re}\{\Phi(x, y) \exp (-2 \mathrm{i} \omega \Omega \mathrm{t})\},
$$

so $\Phi(x, y)$ gives the spatial structure of the flow and $\omega$ its nondimensional frequency. Then $\Phi$ satisfies

$$
\begin{array}{r}
\frac{1}{H} \Delta \Phi+\operatorname{grad}\left(\frac{1}{H}\right) \cdot \operatorname{grad} \Phi+\frac{i}{\omega} \mathbf{k} \cdot\left(\operatorname{grad} \Phi \times \operatorname{grad}\left(\frac{1}{H}\right)\right)=0, \\
\Phi=0 \text { at the coast, } \\
\hat{\mathbf{n}} \cdot \operatorname{grad} \Phi=0 \text { at the shelf-ocean boundary, }
\end{array}
$$

where vector $\hat{\mathbf{n}}$ is normal to the shelf-ocean boundary.

Mathematically, we are going to study the existence of trapped modes (i.e., the eigenvalues either embedded into the essential spectrum or lying in the gap of the essential spectrum) for the problem (1.8)-(1.10) in a curved strip. Similar problems for the Laplace operator have been extensively studied in the literature -ither in a curved strip, in a straight strip with an obstacle, or in a strip with compactly perturbed boundary. In the case of Laplacians with Dirichlet boundary conditions these problems are usually called "quantum waveguides"; the Neumann case is usually referred to as "acoustic waveguides." The important result concerning quantum 
waveguides was established in [ExSe], [DuEx]: in the curved waveguides there always exists a trapped mode. Later this result was extended to more general settings; in particular, in [DiKr] (see also $[\mathrm{KrKr}]$ ) it was shown that in the case of mixed boundary conditions (i.e., Dirichlet conditions on one side of the strip and Neumann conditions on the other side) trapped modes exist if the strip is curved "in the direction of the Dirichlet boundary."

The case of acoustic waveguides is more complicated because any eventual eigenvalues are embedded into the essential spectrum and are, therefore, highly unstable. Therefore, it is believed that in general the existence of trapped modes in this case is due to some sort of the symmetry of the problem (see [EvLeVa], [DaPa], [AsPaVa]).

In the present paper we use an approach similar to the one used in [DuEx] and [EvLeVa]; however, we have to modify this approach substantially due to the fact that we are working with a spectral problem for an operator pencil rather than that for an ordinary operator.

The rest of the paper is organized in the following way. In section 2 , we discuss the rigorous mathematical statement of the problem; in section 3 , we study the essential spectrum; and in section 4, we state and prove the main result on the existence of a discrete spectrum (Theorem 4.1). In particular we show that a trapped mode always exists if all of the following conditions are satisfied: (a) the depth profile $H$ does not depend upon the longitudinal coordinate and is monotone increasing and logarithmically concave in the direction perpendicular to the coast; (b) the channel is curved in the direction of the Dirichlet boundary; (c) the curvature is sufficiently small.

Similar results can be obtained in a straight strip if the depth profile $H$ depends nontrivially upon the longitudinal coordinate; we however do not discuss this problem here.

\section{Mathematical statement of the problem.}

2.1. Geometry. The original geometry is a straight planar strip of width $\delta$ :

$$
G_{0}=\{(x, y): x \in \mathbb{R}, y \in(0, \delta)\} .
$$

Deformed geometry $G$ is assumed to be a curved planar strip of constant width $\delta$. To describe it precisely, we introduce the curve $\Gamma=\{(x=X(\xi), y=Y(\xi))\}, \xi \in \mathbb{R}$, where $\xi$ is a natural arc-length parameter, i.e., $X^{\prime}(\xi)^{2}+Y^{\prime}(\xi)^{2} \equiv 1$. By

$$
\gamma(\xi)=X^{\prime \prime}(\xi) Y^{\prime}(\xi)-X^{\prime}(\xi) Y^{\prime \prime}(\xi)
$$

we denote a (signed) curvature of $\Gamma$ (see Figure 1 and Remark 2.2). Note that $|\gamma(\xi)|^{2}=$ $X^{\prime \prime}(\xi)^{2}+Y^{\prime \prime}(\xi)^{2}$.

We additionally assume

$$
\operatorname{supp} \gamma \Subset[-R, R] \quad \text { for some } R>0,
$$

and set

$$
\kappa^{+}=\sup _{\xi \in[-R, R]} \gamma(\xi), \quad \kappa^{-}=-\inf _{\xi \in[-R, R]} \gamma(\xi) .
$$

We shall assume throughout the paper the smoothness condition

$$
\gamma \in C^{\infty}(\mathbb{R})
$$

which can be obviously softened. 


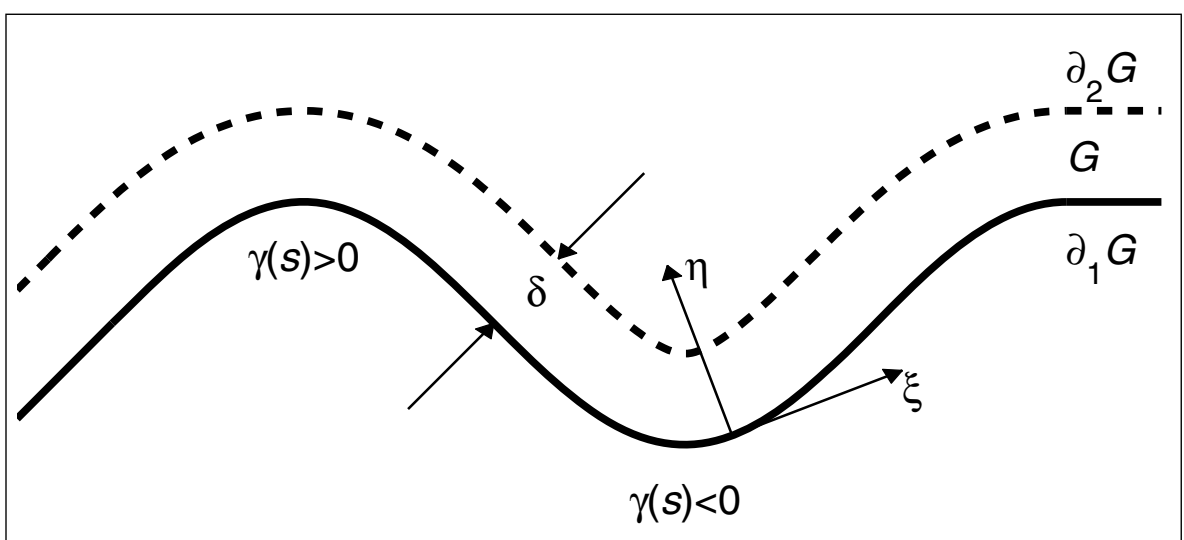

FIG. 1. Domain $G$ and curvilinear coordinates $\xi$, $\eta$. The solid line denotes the boundary $\partial_{1} G$ with the Dirichlet boundary condition, and the dotted line denotes the boundary $\partial_{2} G$ with the Neumann boundary condition. as

Now we can introduce, in a neighborhood of $\Gamma$, the curvilinear coordinates $(\xi, \eta)$

$$
x=X(\xi)-\eta Y^{\prime}(\xi), \quad y=Y(\xi)+\eta X^{\prime}(\xi),
$$

(where $\eta$ is a distance from a point $(x, y)$ to $\Gamma$ ) and describe the deformed strip $G$ in these coordinates as

$$
G=G_{\gamma}=\{(\xi, \eta): \xi \in \mathbb{R}, \eta \in(0, \delta)\}
$$

REMARK 2.1. As sets of points, $G_{\gamma} \equiv G_{0}$ for any $\gamma$, but the metrics are different, see below. We shall often omit the index $\gamma$ if the metric is obvious from the context.

REMARK 2.2. Often one chooses the opposite sign in the definition of the signed curvature $\gamma$ in (2.1). Our choice, though not canonical, is made to match the one in [ExSe].

To avoid local self-intersections, we must restrict the width of the strip by natural conditions

$$
\kappa^{ \pm} \leq A \delta^{-1}, \quad A=\text { const } \in[0,1) .
$$

We shall also assume throughout, without stating it explicitly, that $G$ does not selfintersect globally, i.e., the mapping $(\xi, \eta) \mapsto(x, y)$ given by $(2.5)$ is an injection on $G$.

Finally, it is an easy computation to show that the Euclidean metric in the curvilinear coordinates has a form $d x^{2}+d y^{2}=g d \xi^{2}+d \eta^{2}$, where

$$
g(\xi, \eta)=(1+\eta \gamma(\xi))^{2} .
$$

Later on, we shall widely use the notation

$$
p(\xi, \eta)=(g(\xi, \eta))^{1 / 2}=1+\eta \gamma(\xi) .
$$

Note that in all the volume integrals,

$$
\mathrm{d} G_{\gamma}=p(\xi, \eta) \mathrm{d} \xi \mathrm{d} \eta=(1+\eta \gamma(\xi)) \mathrm{d} \xi \mathrm{d} \eta=p(\xi, \eta) \mathrm{d} G_{0}
$$


2.2. Governing equations. For a given positive continuously differentiable function $H(\xi, \eta)$ (describing a depth profile), we are looking for a function $\Phi(\xi, \eta)$ satisfying (1.8) with spectral parameter $\omega$.

By substituting

$$
\beta(\xi, \eta):=\ln H(\xi, \eta)
$$

and using explicit expressions for differential operators in curvilinear coordinates, we can rewrite (1.8) as

$$
\begin{aligned}
& \omega\left(-\frac{1}{p^{2}} \frac{\partial^{2} \Phi}{\partial \xi^{2}}-\frac{\partial^{2} \Phi}{\partial \eta^{2}}+\left(\frac{1}{p^{3}} \frac{\partial p}{\partial \xi}+\frac{1}{p^{2}} \frac{\partial \beta}{\partial \xi}\right) \frac{\partial \Phi}{\partial \xi}+\left(\frac{\partial \beta}{\partial \eta}-\frac{1}{p} \frac{\partial p}{\partial \eta}\right) \frac{\partial \Phi}{\partial \eta}\right) \\
& =\frac{i}{p}\left(\frac{\partial \beta}{\partial \xi} \frac{\partial \Phi}{\partial \eta}-\frac{\partial \beta}{\partial \eta} \frac{\partial \Phi}{\partial \xi}\right) .
\end{aligned}
$$

REMARK 2.3. When deducing (2.10), we have cancelled, on both sides, a common positive factor $h(\xi, \eta):=\frac{1}{H(\xi, \eta)}=\mathrm{e}^{-\beta(\xi, \eta)}$. However, we have to use this factor when considering corresponding variational equations, in order to keep the resulting forms symmetric. This leads to a special choice of weighted Hilbert spaces below.

Further on, we consider only the case of a longitudinally uniform monotone depth profile,

$$
\beta(\xi, \eta) \equiv \beta(\eta), \quad \beta^{\prime}(\eta)>0,
$$

in which case (2.10) simplifies to

$$
\begin{aligned}
& \omega\left(-\frac{1}{p^{2}} \frac{\partial^{2} \Phi}{\partial \xi^{2}}-\frac{\partial^{2} \Phi}{\partial \eta^{2}}+\frac{1}{p^{3}} \frac{\partial p}{\partial \xi} \frac{\partial \Phi}{\partial \xi}+\left(\beta^{\prime}-\frac{1}{p} \frac{\partial p}{\partial \eta}\right) \frac{\partial \Phi}{\partial \eta}\right) \\
& =-\frac{i}{p} \beta^{\prime} \frac{\partial \Phi}{\partial \xi}
\end{aligned}
$$

with $\beta^{\prime}=\frac{\mathrm{d} \beta}{\mathrm{d} \eta}$.

2.3. Boundary conditions. Let $\partial_{1} G=\{(\xi, 0): \xi \in \mathbb{R}\}$ and $\partial_{2} G=\{(\xi, \delta)$ : $\xi \in \mathbb{R}\}$ denote the lower and the upper boundary of the strip $G$, respectively. Boundary conditions (1.9), (1.10) then become

$$
\left.\Phi\right|_{\partial_{1} G}=\left.\frac{\partial \Phi}{\partial \eta}\right|_{\partial_{2} G}=0
$$

REMARK 2.4. If the flow is confined to a channel, then the Dirichlet boundary condition (1.9) applies on both channel walls. This leads to a mathematically different problem which we do not consider in this paper.

2.4. Function spaces and rigorous operator statement. We want to discuss the function spaces in which everything acts. Let us denote by $L_{2}(G ; h)$ the Hilbert space of functions $\phi: G \rightarrow \mathbb{C}$ which are square-integrable on $G$ with the weight $h(\eta) \equiv \frac{1}{H}=\exp (-\beta(\eta))$ :

$$
\|\phi\|_{L_{2}(G ; h)}^{2}=\int_{G}|\phi(\xi, \eta)|^{2} h(\eta) \mathrm{d} G=\int_{\mathbb{R}} \int_{0}^{\delta}|\phi(\xi, \eta)|^{2} h(\eta) p(\xi, \eta) \mathrm{d} \eta \mathrm{d} \xi<\infty .
$$


The corresponding inner product will be denoted $\langle\cdot, \cdot\rangle_{L_{2}(G ; h)}$. Similarly we can define the space $L_{2}(F ; h)$ for an arbitrary open subset $F$ of $G$.

Let us formally introduce the operators

$$
\mathcal{L}_{\gamma}: \Phi \mapsto-\frac{1}{p^{2}} \frac{\partial^{2} \Phi}{\partial \xi^{2}}-\frac{\partial^{2} \Phi}{\partial \eta^{2}}+\frac{1}{p^{3}} \frac{\partial p}{\partial \xi} \frac{\partial \Phi}{\partial \xi}+\left(\beta^{\prime}-\frac{1}{p} \frac{\partial p}{\partial \eta}\right) \frac{\partial \Phi}{\partial \eta}
$$

and

$$
\mathcal{M}_{\gamma}: \Phi \mapsto-\frac{i}{p} \beta^{\prime} \frac{\partial \Phi}{\partial \xi}
$$

(The dependence on $\gamma$ is of course via $p$; see (2.8).) Then (2.12) can be formally rewritten as

$$
\omega \mathcal{L}_{\gamma} \Phi=\mathcal{M}_{\gamma} \Phi
$$

or via an operator pencil

$$
\mathcal{A}_{\gamma} \equiv \mathcal{A}_{\gamma}(\omega)=\omega \mathcal{L}_{\gamma}-\mathcal{M}_{\gamma}
$$

as

$$
\mathcal{A}_{\gamma}(\omega) \Phi=0
$$

The domain of the pencil $\mathcal{A}_{\gamma}$ in the $L_{2}$-sense is naturally defined as

$$
\operatorname{Dom}\left(\mathcal{A}_{\gamma}\right)=\left\{\Phi \in H^{2}(G), \Phi \text { satisfies }(2.13)\right\},
$$

where $H^{2}$ denotes a standard Sobolev space.

On the domain (2.17), $\mathcal{M}_{\gamma}$ is symmetric, and $\mathcal{L}_{\gamma}$ is symmetric and positive in the sense of the scalar product $\langle\cdot, \cdot\rangle_{L_{2}(G ; h)}$, with

$$
\left\langle\mathcal{L}_{\gamma} \Phi, \Phi\right\rangle_{L_{2}(G ; h)}=\int_{\mathbb{R}} \int_{0}^{\delta}\left(\frac{1}{p}\left|\frac{\partial \Phi}{\partial \xi}\right|^{2}+p\left|\frac{\partial \Phi}{\partial \eta}\right|^{2}\right) \mathrm{e}^{-\beta} \mathrm{d} \eta \mathrm{d} \xi .
$$

Later on, we shall use a weak (or variational) form of (2.16), and shall require some other function spaces described below. Let $F \subseteq G$, and suppose its boundary is decomposed into two disjoint parts: $\partial F=\partial_{1} F \sqcup \partial_{2} F$. We introduce the space

$$
\begin{aligned}
\widetilde{C}_{0}^{\infty}\left(F ; \partial_{1} F\right) & =\left\{\phi \in C^{\infty}(F): \overline{\operatorname{supp} \phi} \cap \partial_{1} F=\emptyset\right. \\
& \text { and there exists } r>0 \text { such that } \phi(\xi, \eta)=0 \text { for }(\xi, \eta) \in F,|\xi| \geq r\}
\end{aligned}
$$

consisting of smooth functions with compact support vanishing near $\partial_{1} F$.

By $\widetilde{H}_{0}^{1}\left(F ; \partial_{1} F ; h\right)$ we denote the closure of $\widetilde{C}_{0}^{\infty}\left(F ; \partial_{1} F\right)$ with respect to the scalar product

$$
\langle\phi, \psi\rangle_{\widetilde{H}_{0}^{1}\left(F, \partial_{1} F ; h\right)}=\langle\phi, \psi\rangle_{L_{2}(F ; h)}+\langle\operatorname{grad} \phi, \operatorname{grad} \psi\rangle_{L_{2}(F ; h)} .
$$

In what follows we shall study the operators $\mathcal{L}_{\gamma}, \mathcal{M}_{\gamma}$ and the pencil $\mathcal{A}_{\gamma}$ from a variational point of view. The details are given in the next section; here we note only that from now we understand the expression $\left\langle\mathcal{L}_{\gamma} \Psi, \Psi\right\rangle_{L_{2}\left(G_{\gamma}, h\right)}$ as the quadratic form for the operator $\mathcal{L}_{\gamma}$, with the quadratic form domain $\widetilde{H}_{0}^{1}\left(G ; \partial_{1} G ; h\right)$. 
The main purpose of this paper is to study the spectral properties of the operator pencil $\mathcal{A}_{\gamma}$. We recall the following definitions.

A number $\omega \in \mathbb{C}$ is said to belong to the spectrum of $\mathcal{A}_{\gamma}\left(\operatorname{denoted} \operatorname{spec}\left(\mathcal{A}_{\gamma}\right)\right)$ if $\mathcal{A}_{\gamma}(\omega)$ is not invertible.

It is easily seen that in our case the spectrum of $\mathcal{A}_{\gamma}$ is real.

We say that $\omega \in \mathbb{R}$ belongs to the essential spectrum of the operator pencil $\mathcal{A}_{\gamma}$ (denoted $\omega \in \operatorname{spec}_{\text {ess }}\left(\mathcal{A}_{\gamma}\right)$ ) if for this $\omega$ the operator $\mathcal{A}_{\gamma}(\omega)$ is non-Fredholm.

We say that $\omega \in \mathbb{C}$ belongs to the point spectrum of the operator pencil $\mathcal{A}_{\gamma}$ (denoted $\omega \in \operatorname{spec}_{\mathrm{pt}}\left(\mathcal{A}_{\gamma}\right)$ ), or, in other words, say that $\omega$ is an eigenvalue, if for this $\omega$ there exists a nontrivial solution $\Psi \in \operatorname{Dom}\left(\mathcal{A}_{\gamma}\right)$ of the problem $\mathcal{A}_{\gamma}(\omega) \Psi=0$.

It is known that the essential spectrum is a closed subset of $\mathbb{R}$, and that any point of the spectrum outside the essential spectrum is an isolated eigenvalue of finite multiplicity. The set of all such points is called the discrete spectrum, and will be denoted $\operatorname{spec}_{\text {dis }}\left(\mathcal{A}_{\gamma}\right)$. There may, however, exist the points of the spectrum which belong to both the essential spectrum and the point spectrum.

Our main result (Theorem 4.1 below) establishes some conditions on the curvature $\gamma$ of the waveguide which guarantee the existence of eigenvalues of $\mathcal{A}_{\gamma}$.

It is more convenient to deal with problems of this type variationally, and we start the next section with an abstract variational scheme suitable for self-adjoint pencils with nonempty essential spectrum.

\section{Essential spectrum.}

3.1. Variational principle for the essential spectrum.

Definition 3.1. We set, for $j \in \mathbb{N}$,

$$
\mu_{\gamma, j}=\sup _{\substack{U \subset \widetilde{H}_{0}^{1}\left(G ; \partial_{1} G ; h\right) \\ \operatorname{dim} U=j}} \inf _{\Psi \in U, \Psi \neq 0} \frac{\left\langle\mathcal{M}_{\gamma} \Psi, \Psi\right\rangle_{L_{2}\left(G_{\gamma}, h\right)}}{\left\langle\mathcal{L}_{\gamma} \Psi, \Psi\right\rangle_{L_{2}\left(G_{\gamma}, h\right)}} .
$$

As $\left\langle\mathcal{L}_{\gamma} \Psi, \Psi\right\rangle_{L_{2}\left(G_{\gamma}, h\right)}$ is positive, the right-hand side of (3.1) is well defined, though the numbers $\mu_{\gamma, j}$ may a priori be finite or infinite.

Obviously, for any fixed curvature profile $\gamma$ the numbers $\mu_{\gamma, j}$ form a nonincreasing sequence:

$$
\mu_{\gamma, 1} \geq \mu_{\gamma, 2} \geq \cdots \geq \mu_{\gamma, j} \geq \mu_{\gamma, j+1} \geq \cdots .
$$

Definition 3.2. Denote

$$
\underline{\mu}_{\gamma}=\lim _{j \rightarrow \infty} \mu_{\gamma, j}
$$

For general self-adjoint operator pencils the analogue of (3.2) may be finite or equal to $\pm \infty$; as we shall see below, in our case $\mu_{\gamma}$ is finite.

The following result is a modification, to the case of an abstract self-adjoint linear pencil, of the general variational principle for a self-adjoint operator with an essential spectrum; see [Da, Prop. 4.5.2].

Proposition 3.3. Either

or

(i) $\underline{\mu}_{\gamma}>-\infty$, and then $\sup \operatorname{spec}_{\text {ess }}\left(\mathcal{A}_{\gamma}\right)=\underline{\mu}_{\gamma}$,

(ii) $\underline{\mu}_{\gamma}=-\infty$, and then $\operatorname{spec}_{\mathrm{ess}}\left(\mathcal{A}_{\gamma}\right)=\emptyset$. Moreover, if $\mu_{\gamma, j}>\underline{\mu}_{\gamma}$, then $\mu_{\gamma, j} \in \operatorname{spec}_{\text {dis }}\left(\mathcal{A}_{\gamma}\right)$.

Proposition 3.3 ensures that we can use the variational principle (3.1) in order to find the eigenvalues of the pencil $\mathcal{A}_{\gamma}$ lying above the supremum $\underline{\mu}_{\gamma}$ of the essential spectrum. 
3.2. Essential spectrum for the straight strip. The spectral analysis in the case of a straight strip $(\gamma \equiv 0)$ is rather straightforward as the problem admits in this case the separation of variables.

Let us seek the solutions of (2.12), (2.13) in the case of a straight strip $(\gamma \equiv 0$, and so $p \equiv 1$ ) in the form

$$
\Phi(\xi, \eta)=\phi(\eta) \exp (i \alpha \xi)
$$

it is sufficient to consider only real values of $\alpha$.

After separation of variables, (2.12), (2.13) are written, for each $\alpha$, as a onedimensional transversal spectral problem

$$
\omega\left(-\phi^{\prime \prime}+\beta^{\prime} \phi^{\prime}+\alpha^{2} \phi\right)=\alpha \beta^{\prime} \phi, \quad \phi(0)=\phi^{\prime}(\delta)=0 .
$$

Alternatively, introduce operators

$$
\mathfrak{l}_{\alpha}: \phi \mapsto-\phi^{\prime \prime}+\beta^{\prime} \phi^{\prime}+\alpha^{2} \phi, \quad \mathfrak{m}_{\alpha}: \phi \mapsto \alpha \beta^{\prime} \phi,
$$

and a pencil

$$
\mathfrak{a}_{\alpha}(\omega)=\omega \mathfrak{l}_{\alpha}-\mathfrak{m}_{\alpha},
$$

(again understood in an $L_{2}((0, \delta) ; h)$ sense with the domain defined similarly to $(2.17)$ ), and consider a one-dimensional operator pencil spectral problem $\mathfrak{a}_{\alpha}(\omega) \phi=0$.

For a fixed value of $\alpha$, the one-dimensional linear operator pencil (3.4) has the essential spectrum $\{0\}$ and a discrete $\operatorname{spectrum~} \operatorname{spec}\left(\mathfrak{a}_{\alpha}\right)$; note that

$$
\operatorname{spec}\left(\mathfrak{a}_{-\alpha}\right)=-\operatorname{spec}\left(\mathfrak{a}_{\alpha}\right) .
$$

Denote, for $\alpha>0$, the top of the spectrum of this transversal problem by $\omega_{\alpha}=$ $\sup \operatorname{spec}\left(\mathfrak{a}_{\alpha}\right)$.

Lemma 3.4. Let $\alpha>0$. Then, under condition (2.11),

(i) $\operatorname{spec}\left(\mathfrak{a}_{\alpha}\right) \subset[0,+\infty)$;

(ii) $0<\omega_{\alpha}<+\infty$;

(iii) $\omega_{\alpha} \rightarrow+0$ as $\alpha \rightarrow \infty$.

Proof. By the variational principle analogous to Proposition 3.3(i),

$$
\omega_{\alpha}=\sup _{\substack{\phi \in \widetilde{H}_{0}^{1}((0, \delta), 0, h) \\ \phi \neq 0}} J_{\alpha}(\phi)
$$

where we set

$$
J_{\alpha}(\phi)=\frac{\left\langle\mathfrak{m}_{\alpha} \phi, \phi\right\rangle_{L_{2}((0, \delta), h)}}{\left\langle\mathfrak{l}_{\alpha} \phi, \phi\right\rangle_{L_{2}((0, \delta), h)}}=\frac{\int_{0}^{\delta} \alpha \beta^{\prime}(\eta) \phi(\eta)^{2} h(\eta) \mathrm{d} \eta}{\int_{0}^{\delta}\left(-\phi^{\prime \prime}(\eta)+\beta^{\prime}(\eta) \phi^{\prime}(\eta)+\alpha^{2} \phi(\eta)\right) \phi(\eta) h(\eta) \mathrm{d} \eta} .
$$

After integrating by parts using $h(\eta)=\mathrm{e}^{-\beta(\eta)}$ and inverting the quotient, we get

$$
J_{\alpha}(\phi)=\left(\alpha J^{(1)}(\phi)+\frac{1}{\alpha} J^{(2)}(\phi)\right)^{-1}
$$


where we denote

$$
J^{(1)}(\phi)=\frac{\int_{0}^{\delta} \mathrm{e}^{-\beta(\eta)}|\phi(\eta)|^{2} \mathrm{~d} \eta}{\int_{0}^{\delta} \beta^{\prime}(\eta) \mathrm{e}^{-\beta(\eta)}|\phi(\eta)|^{2} \mathrm{~d} \eta}
$$

and

$$
J^{(2)}(\phi)=\frac{\int_{0}^{\delta} \mathrm{e}^{-\beta(\eta)}\left|\phi^{\prime}(\eta)\right|^{2} \mathrm{~d} \eta}{\int_{0}^{\delta} \beta^{\prime}(\eta) \mathrm{e}^{-\beta(\eta)}|\phi(\eta)|^{2} \mathrm{~d} \eta} .
$$

The statements (ii) and (iii) of the lemma now follow immediately from the estimates

$$
J^{(1)}(\phi) \geq \frac{\inf _{\eta \in(0, \delta)} \mathrm{e}^{-\beta(\eta)}}{\sup _{\eta \in(0, \delta)}\left(\beta^{\prime}(\eta) \mathrm{e}^{-\beta(\eta)}\right)}
$$

and

$$
J^{(2)}(\phi) \geq \frac{\pi^{2}}{4 \delta^{2}} \frac{\inf _{\eta \in(0, \delta)} \mathrm{e}^{-\beta(\eta)}}{\sup _{\eta \in(0, \delta)}\left(\beta^{\prime}(\eta) \mathrm{e}^{-\beta(\eta)}\right)},
$$

where the latter inequality uses the variational principle and the fact that the principal eigenvalue of the mixed Dirichlet-Neumann spectral problem for the operator $-\frac{\mathrm{d}^{2}}{\mathrm{~d} \eta^{2}}$ on the interval $(0, \delta)$ is equal to $\frac{\pi^{2}}{4 \delta^{2}}$. The statement (i) follows from the positivity of the right-hand side of (3.7).

We are now able to find the essential spectrum of the problem in a straight strip.

Lemma 3.5. Assume that conditions (2.11) hold. Then

$$
\operatorname{spec}_{\text {ess }}\left(\mathcal{A}_{0}\right)=\left[-\Omega_{*}, \Omega_{*}\right]
$$

where

$$
\Omega_{*}=\sup _{\phi \in \widetilde{H}_{0}^{1}((0, \delta), 0, h)} \frac{\frac{1}{2} \int_{0}^{\delta} \beta^{\prime}(\eta) \mathrm{e}^{-\beta(\eta)}|\phi(\eta)|^{2} \mathrm{~d} \eta}{\sqrt{\int_{0}^{\delta} \mathrm{e}^{-\beta(\eta)}\left|\phi^{\prime}(\eta)\right|^{2} \mathrm{~d} \eta \cdot \int_{0}^{\delta} \mathrm{e}^{-\beta(\eta)}|\phi(\eta)|^{2} \mathrm{~d} \eta}}>0 .
$$

Proof. It is standard that

$$
\operatorname{spec}_{\mathrm{ess}}\left(\mathcal{A}_{0}\right)=\overline{\bigcup_{\alpha \in \mathbb{R}} \operatorname{spec}\left(\mathfrak{a}_{\alpha}\right)}
$$

Thus, by Lemma 3.4, and with account of the antisymmetry of the spectrum of $\mathfrak{a}_{\alpha}$ with respect to $\alpha$ and its positivity for $\alpha>0$, we have

$$
\sup \operatorname{spec}_{\text {ess }}\left(\mathcal{A}_{0}\right)=\sup _{\alpha>0} \omega_{\alpha}=\sup _{\alpha>0} \sup _{\phi \in \widetilde{H}_{0}^{1}((0, \delta), 0, h)} J_{\alpha}(\phi) .
$$


By maximizing first with respect to $\alpha$, we obtain, from (3.7),

$$
J_{\alpha}(\phi) \leq J_{\alpha_{*}(\phi)}(\phi)
$$

with the maximizer

$$
\alpha_{*}(\phi)=\sqrt{\frac{J^{(2)}(\phi)}{J^{(1)}(\phi)}} .
$$

Maximizing now with respect to $\phi$ gives $\sup \operatorname{spec}_{\text {ess }}\left(\mathcal{A}_{0}\right)=\Omega_{*}$, with $\Omega_{*}$ given by (3.9). Finally, we note that $\omega_{\alpha}$ depends continuously on $\alpha>0$. Since $\omega_{\alpha} \rightarrow+0$ as $\alpha \rightarrow+\infty$ by Lemma 3.4(iii), $\omega_{\alpha}$ thus takes all the values in $\left(0, \Omega_{*}\right]$. Therefore, the closed interval $\left[0, \Omega_{*}\right]$ lies in $\operatorname{spec}_{\text {ess }}\left(\mathcal{A}_{0}\right)$. By symmetry $(3.5)$, we also have $\left[-\Omega_{*}, 0\right] \subset$ $\operatorname{spec}_{\text {ess }}\left(\mathcal{A}_{0}\right)$, which finishes the proof.

3.3. Essential spectrum for a curved strip. It is now a standard procedure to show that under our conditions the essential spectrum of the problem in a curved strip coincides with the essential spectrum of the problem in a straight strip given by Lemma 3.5. Namely, we have the following.

Lemma 3.6. Let us assume conditions (2.2), (2.4), and (2.11) hold. Then

$$
\operatorname{spec}_{\text {ess }}\left(\mathcal{A}_{\gamma}\right)=\operatorname{spec}_{\text {ess }}\left(\mathcal{A}_{0}\right)=\left[-\Omega_{*}, \Omega_{*}\right]
$$

with $\Omega_{*}$ given by (3.9).

The proof is based on the fact that any solution of the problem $(2.12),(2.13)$ with $\gamma \not \equiv 0$ (and thus $p \not \equiv 1$ ) should coincide in

$$
G \cap\{|\xi|>R>\max (|\inf \operatorname{supp} \gamma|,|\sup \operatorname{supp} \gamma|)\}
$$

with a solution of the same problem for $\gamma \equiv 0$. An analogous result has been proved in a number of similar situations elsewhere (see, e.g., [ExSe], [EvLeVa], [DaPa], [KrTA]), so we omit the details of the proof. We briefly note that the inclusion $\operatorname{spec}_{\text {ess }}\left(\mathcal{A}_{\gamma}\right) \subseteq$ $\operatorname{spec}_{\text {ess }}\left(\mathcal{A}_{0}\right)$ is proved using the separation of variables as above and a construction of appropriate Weyl's sequences, and in order to prove the inclusion $\operatorname{spec}_{\text {ess }}\left(\mathcal{A}_{\gamma}\right) \supseteq$ $\operatorname{spec}_{\text {ess }}\left(\mathcal{A}_{0}\right)$ one can use the Dirichlet-Neumann bracketing and the discreteness of the spectrum of the problem (2.12), (2.13) considered in $G \cap\{|\xi|<R\}$ with additional Dirichlet or Neumann boundary conditions imposed on the "cuts" $\{\xi= \pm R\}$.

4. Main result. Our main result consists in stating some sufficient conditions on the depth profile $\beta(\eta)$ and the curvature profile $\gamma(\xi)$ which guarantee the existence of an eigenvalue of the pencil $\mathcal{A}_{\gamma}$ lying outside the essential spectrum.

Theorem 4.1. Assume, as before, that condition (2.11) holds. Assume additionally that

$$
\beta^{\prime \prime}(\eta)<0 \quad \text { for } \eta \in(0, \delta) .
$$

Then there exists a constant $C_{\beta}>0$, which depends only on the depth profile $\beta$, such that $\operatorname{spec}_{\mathrm{dis}}\left(\mathcal{A}_{\gamma}\right) \neq \emptyset$ whenever $\gamma$ satisfies conditions (2.2), (2.4), and

$$
\int \gamma(\xi) \mathrm{d} \xi>C_{\beta} \int \gamma(\xi)^{2} \mathrm{~d} \xi
$$

We give an explicit expression for $C_{\beta}$ below; see (4.15). 
An integral sufficient condition (4.2) may be replaced by a pointwise, although more restrictive, condition.

Corollary 4.2. Assume that conditions (2.11) and (4.1) hold. Then there exists a constant $c_{\beta, R}=\frac{C_{\beta}}{2 R}$ which depends only on the depth profile $\beta$ and a given $R>0$ such that $\operatorname{spec}_{\mathrm{dis}}\left(\mathcal{A}_{\gamma}\right) \neq \emptyset$ whenever $\gamma \not \equiv 0$ satisfies conditions (2.2), (2.4), and

$$
0 \leq \gamma(\xi)<c_{\beta, R} \quad \text { for }|\xi| \leq R .
$$

We prove Theorem 4.1 using a number of simple lemmas, the central of which is the following.

Lemma 4.3. Suppose there exists a function $\widetilde{\Psi} \in \widetilde{H}_{0}^{1}\left(G_{\gamma}, h\right)$ such that

$$
\frac{\left\langle\mathcal{M}_{\gamma} \widetilde{\Psi}, \widetilde{\Psi}\right\rangle_{L_{2}\left(G_{\gamma}, h\right)}}{\left\langle\mathcal{L}_{\gamma} \widetilde{\Psi}, \widetilde{\Psi}\right\rangle_{L_{2}\left(G_{\gamma}, h\right)}}>\Omega_{*} .
$$

Then there exists $\omega>\Omega_{*}$ which belongs to $\operatorname{spec}_{\mathrm{dis}}\left(\mathcal{A}_{\gamma}\right)$.

Lemma 4.3 is just a restatement of the variational principle of Proposition 3.3. The main difficulty in its application is of course the choice of an appropriate test function $\widetilde{\Psi}$. However such choice becomes much easier if we use the following modification of this lemma which allows us to consider test functions which are not necessarily square-integrable on $G_{\gamma}$.

Denote, for brevity, $G_{\gamma}^{r}=G_{\gamma} \cap\{|\xi|<r\}$.

Lemma 4.4. Suppose there exist a function $\Psi$ and a constant $D$ such that, for any $r>R$, we have $\Psi \in \widetilde{H}_{0}^{1}\left(G_{\gamma}^{r}, h\right)$ and

$$
\left\langle\mathcal{M}_{\gamma} \Psi, \Psi\right\rangle_{L_{2}\left(G_{\gamma}^{r}, h\right)}-\Omega_{*}\left\langle\mathcal{L}_{\gamma} \Psi, \Psi\right\rangle_{L_{2}\left(G_{\gamma}^{r}, h\right)} \geq D>0
$$

Then there exists $\omega>\Omega_{*}$ which belongs to $\operatorname{spec}_{\mathrm{dis}}\left(\mathcal{A}_{\gamma}\right)$.

The proof of Lemma 4.4 uses the construction of an appropriate cutoff function $\chi(\xi)$ such that $\widetilde{\Psi}=\chi \Psi$ satisfies the conditions of Lemma 4.3; cf. [DaPa, Prop. 1].

We now proceed as follows.

Let $\phi_{*}(\eta)$ be a maximizer in (3.9), and set

$$
\Psi(\xi, \eta)=\phi_{*}(\eta) \mathrm{e}^{i \alpha \cdot \xi}
$$

where

$$
\alpha_{\bullet}=\alpha_{*}\left(\phi_{*}\right)=\sqrt{\frac{J^{(2)}\left(\phi_{*}\right)}{J^{(1)}\left(\phi_{*}\right)}} .
$$

It is important to note that $\Psi$ is in fact an "eigenfunction" of the essential spectrum of $\mathcal{A}_{\gamma}$ corresponding to its highest positive point $\Omega_{*}$ and that $\phi_{*}$ is an eigenfunction of (3.4) with $\alpha=\alpha_{\bullet}$ (i.e., of the pencil $\mathfrak{a}_{\alpha \bullet}$ ) again corresponding to the eigenvalue $\Omega_{*}$, and so

$$
\phi_{*}^{\prime \prime}=\beta^{\prime} \phi_{*}^{\prime}+\left(\alpha_{\bullet}^{2}-\Lambda_{*} \alpha_{\bullet} \beta^{\prime}\right) \phi_{*}, \quad \phi_{*}(0)=\phi_{*}^{\prime}(\delta)=0
$$

with $\Lambda_{*}:=\frac{1}{\Omega_{*}}($ cf. $(3.4))$. 
For future use, we summarize the relations obtained so far:

$$
\begin{aligned}
\mathcal{L}_{0} \Psi & =\left(-\phi_{*}^{\prime \prime}(\eta)+\beta^{\prime}(\eta) \phi_{*}^{\prime}(\eta)+\alpha_{\bullet} \phi_{*}(\eta)\right) \mathrm{e}^{i \alpha \bullet \xi}=\left(\mathfrak{l}_{\alpha_{\bullet}} \phi_{*}\right) \mathrm{e}^{i \alpha \bullet \xi} \\
\mathcal{M}_{0} \Psi & =\alpha_{\bullet} \beta^{\prime}(\eta) \phi_{*}(\eta) \mathrm{e}^{i \alpha \cdot \xi}=\left(\mathfrak{m}_{\alpha \bullet} \phi_{*}\right) \mathrm{e}^{i \alpha \bullet \xi} \\
\mathcal{L}_{\gamma} \Psi & =\left(-\phi_{*}^{\prime \prime}(\eta)+\left(\beta^{\prime}(\eta)-\frac{1}{p(\xi, \eta)} \frac{\partial p(\xi, \eta)}{\partial \eta}\right) \phi_{*}^{\prime}(\eta)\right. \\
& \left.\quad+\left(\frac{i \alpha \bullet}{p(\xi, \eta)^{3}} \frac{\partial p(\xi, \eta)}{\partial \xi}+\frac{\alpha_{\bullet}^{2}}{p(\xi, \eta)^{2}}\right) \phi_{*}(\eta)\right) \mathrm{e}^{i \alpha \bullet \xi} \\
\mathcal{M}_{\gamma} \Psi & =\frac{\alpha_{\bullet}}{p(\xi, \eta)} \beta^{\prime}(\eta) \phi_{*}(\eta) \mathrm{e}^{i \alpha \xi} \\
p(\xi, \eta) & =1+\gamma(\xi) \eta
\end{aligned}
$$

(with' denoting differentiation with respect to $\eta$ ).

It is important to note that for any $r>0$,

$$
\frac{\left\langle\mathcal{M}_{0} \Psi, \Psi\right\rangle_{L_{2}\left(G_{0}^{r}, h\right)}}{\left\langle\mathcal{L}_{0} \Psi, \Psi\right\rangle_{L_{2}\left(G_{0}^{r}, h\right)}}=\frac{\left\langle\mathfrak{m}_{\alpha_{\bullet}} \phi_{*}, \phi_{*}\right\rangle_{L_{2}((0, \delta), h)}}{\left\langle\mathfrak{l}_{\alpha_{\bullet}} \phi_{*}, \phi_{*}\right\rangle_{L_{2}((0, \delta), h)}}=\Omega_{*}>0
$$

and, as explicit formulae above show,

$$
\begin{aligned}
\left\langle\mathcal{M}_{\gamma} \Psi, \Psi\right\rangle_{L_{2}\left(G_{\gamma}^{r}, h\right)} & =\left\langle\mathcal{M}_{0} \Psi, \Psi\right\rangle_{L_{2}\left(G_{0}^{r}, h\right)}=\alpha \bullet \int_{-r}^{r} \int_{0}^{\delta} \beta^{\prime}(\eta) \mathrm{e}^{-\beta(\eta)}\left|\phi_{*}(\eta)\right|^{2} \mathrm{~d} \eta \mathrm{d} \xi \\
& =2 r \alpha \bullet \int_{0}^{\delta} \beta^{\prime}(\eta) \mathrm{e}^{-\beta(\eta)}\left|\phi_{*}(\eta)\right|^{2} \mathrm{~d} \eta>0 .
\end{aligned}
$$

We want to show that under conditions of Theorem 4.1 and with the choice of $\Psi$ as above, inequality (4.5) holds for any $r>R$.

In view of (4.8), it is enough to show that

$$
D_{\gamma}:=\left\langle\mathcal{L}_{\gamma} \Psi, \Psi\right\rangle_{L_{2}\left(G_{\gamma}^{r}, h\right)}-\left\langle\mathcal{L}_{0} \Psi, \Psi\right\rangle_{L_{2}\left(G_{0}^{r}, h\right)}
$$

is negative for $r>R$.

Explicit substitution gives, after taking into account the formula

$$
\int \frac{1}{p(\xi, \eta)^{2}} \frac{\partial p(\xi, \eta)}{\partial \xi} \mathrm{d} \xi=0
$$

(due to (2.2), with account of (2.8)), the following expression:

$$
D_{\gamma}=\int_{-r}^{r} \int_{0}^{\delta} \gamma(\xi) \eta \mathrm{e}^{-\beta(\eta)}\left|\phi_{*}^{\prime}(\eta)\right|^{2} \mathrm{~d} \eta \mathrm{d} \xi-\int_{-r}^{r} \int_{0}^{\delta} \alpha_{\bullet}^{2} \frac{\eta \gamma(\xi)}{1+\eta \gamma(\xi)} \mathrm{e}^{-\beta(\eta)}\left|\phi_{*}(\eta)\right|^{2} \mathrm{~d} \eta \mathrm{d} \xi .
$$

This, in turn, can be rewritten, using the obvious identity

$$
\frac{\eta \gamma(\xi)}{1+\eta \gamma(\xi)}=\eta \gamma(\xi)-\frac{\eta^{2} \gamma(\xi)^{2}}{1+\eta \gamma(\xi)}
$$

as

$$
\begin{aligned}
D_{\gamma} & =\int_{-r}^{r} \gamma(\xi) \int_{0}^{\delta} \eta \mathrm{e}^{-\beta(\eta)}\left(\left|\phi_{*}^{\prime}(\eta)\right|^{2}-\alpha_{\bullet}^{2}\left|\phi_{*}(\eta)\right|^{2}\right) \mathrm{d} \eta \mathrm{d} \xi \\
& +\alpha_{\bullet}^{2} \int_{-r}^{r} \int_{0}^{\delta} \frac{\eta^{2} \gamma(\xi)^{2}}{1+\eta \gamma(\xi)} \mathrm{e}^{-\beta(\eta)}\left|\phi_{*}(\eta)\right|^{2} \mathrm{~d} \eta \mathrm{d} \xi .
\end{aligned}
$$


We shall deal with the two terms in (4.9) separately.

The first one is more difficult. As (4.6) yields explicitly

$$
\alpha_{\bullet}^{2}=\frac{\int_{0}^{\delta} \mathrm{e}^{-\beta(\eta)}\left|\phi_{*}^{\prime}(\eta)\right|^{2} \mathrm{~d} \eta}{\int_{0}^{\delta} \mathrm{e}^{-\beta(\eta)}\left|\phi_{*}(\eta)\right|^{2} \mathrm{~d} \eta}
$$

we get

$$
\begin{aligned}
& I_{1}:=\int_{0}^{\delta} \eta \mathrm{e}^{-\beta(\eta)}\left(\left|\phi_{*}^{\prime}(\eta)\right|^{2}-\alpha_{\bullet}^{2}\left|\phi_{*}(\eta)\right|^{2}\right) \mathrm{d} \eta \\
&= \frac{1}{\int_{0}^{\delta} \mathrm{e}^{-\beta(\eta)}\left|\phi_{*}(\eta)\right|^{2} \mathrm{~d} \eta} \times\left(\int_{0}^{\delta} \eta \mathrm{e}^{-\beta(\eta)}\left|\phi_{*}^{\prime}(\eta)\right|^{2} \mathrm{~d} \eta \cdot \int_{0}^{\delta} \mathrm{e}^{-\beta(\eta)}\left|\phi_{*}(\eta)\right|^{2} \mathrm{~d} \eta\right. \\
&\left.\quad-\int_{0}^{\delta} \mathrm{e}^{-\beta(\eta)}\left|\phi_{*}^{\prime}(\eta)\right|^{2} \mathrm{~d} \eta \cdot \int_{0}^{\delta} \eta \mathrm{e}^{-\beta(\eta)}\left|\phi_{*}(\eta)\right|^{2} \mathrm{~d} \eta\right) .
\end{aligned}
$$

We want to show that the term in brackets is negative under some reasonable assumptions.

Lemma 4.5. Assume that the conditions of Theorem 4.1 hold. Then $I_{1}<0$.

The proof of Lemma 4.5 uses the following simple fact. ${ }^{1}$

LEMmA 4.6. Let $(a, b) \subset(0,+\infty)$ be a finite interval, and let a function $g$ : $(a, b) \rightarrow \mathbb{R}$ be nonincreasing. Then

$$
\left(\int_{a}^{b} x g(x) f(x) \mathrm{d} x\right) \cdot\left(\int_{a}^{b} f(x) \mathrm{d} x\right)-\left(\int_{a}^{b} g(x) f(x) \mathrm{d} x\right) \cdot\left(\int_{a}^{b} x f(x) \mathrm{d} x\right) \leq 0
$$

for any nonnegative function $f:(a, b) \rightarrow \mathbb{R}$.

Proof of Lemma 4.6. We have

$$
\begin{gathered}
\left(\int_{a}^{b} x g(x) f(x) \mathrm{d} x\right) \cdot\left(\int_{a}^{b} f(x) \mathrm{d} x\right)-\left(\int_{a}^{b} g(x) f(x) \mathrm{d} x\right) \cdot\left(\int_{a}^{b} x f(x) \mathrm{d} x\right) \\
\quad=\int_{a}^{b} \int_{a}^{b} x g(x) f(x) f(y) \mathrm{d} x \mathrm{~d} y-\int_{a}^{b} \int_{a}^{b} g(x) f(x) y f(y) \mathrm{d} x \mathrm{~d} y \\
=\int_{a}^{b} \int_{a}^{y}(x-y) f(x) f(y) g(x) \mathrm{d} x \mathrm{~d} y+\int_{a}^{b} \int_{y}^{b}(x-y) f(x) f(y) g(x) \mathrm{d} x \mathrm{~d} y .
\end{gathered}
$$

Interchanging the variables $x$ and $y$ in the last integral, we obtain that the whole expression is equal to

$$
\int_{a}^{b} \int_{a}^{y} \underbrace{(x-y)}_{\text {nonpositive }} \underbrace{f(x) f(y)(g(x)-g(y))}_{\text {nonnegative }} \mathrm{d} x \mathrm{~d} y
$$

and is therefore nonpositive.

\footnotetext{
${ }^{1}$ We are grateful to Daniel Elton for a useful suggestion that helped to prove this lemma.
} 
We can now proceed with evaluating $I_{1}$.

Proof of Lemma 4.5. We act by doing a lot of integrations by parts. We shall also use (4.7).

We have (all integrals are over $[0, \delta]$ and with respect to $\eta$ )

$$
\begin{aligned}
\int \eta \mathrm{e}^{-\beta}\left|\phi_{*}^{\prime}\right|^{2} & =-\int \phi_{*} \cdot\left(\eta \mathrm{e}^{-\beta} \phi_{*}^{\prime}\right)^{\prime} \\
& =-\int \phi_{*} \cdot\left(\mathrm{e}^{-\beta} \phi_{*}^{\prime}-\beta^{\prime} \eta \mathrm{e}^{-\beta} \phi_{*}^{\prime}+\eta \mathrm{e}^{-\beta} \phi_{*}^{\prime \prime}\right) \\
& =-\int \phi_{*} \cdot\left(\mathrm{e}^{-\beta} \phi_{*}^{\prime}+\left(\alpha_{\bullet}^{2}-\Lambda_{*} \alpha_{\bullet} \beta^{\prime}\right) \mathrm{e}^{-\beta} \eta \phi_{*}\right) .
\end{aligned}
$$

Further,

$$
-\int\left(\phi_{*} \mathrm{e}^{-\beta}\right) \phi_{*}^{\prime}=\left(\int\left(\phi_{*}^{\prime} \mathrm{e}^{-\beta}-\beta^{\prime} \phi_{*} \mathrm{e}^{-\beta}\right) \phi_{*}\right)-\mathrm{e}^{-\beta(\delta)} \phi_{*}^{2}(\delta),
$$

thus producing

$$
\begin{aligned}
\int \eta \mathrm{e}^{-\beta}\left|\phi_{*}^{\prime}\right|^{2} & =-\frac{1}{2} \int \beta^{\prime} \phi_{*}^{2} \mathrm{e}^{-\beta} \underbrace{-\frac{1}{2} \mathrm{e}^{-\beta(\delta)} \phi_{*}^{2}(\delta)}_{\text {negative constant }} \\
& -\alpha_{\bullet}^{2} \int \eta \mathrm{e}^{-\beta}\left|\phi_{*}\right|^{2}+\Lambda_{*} \alpha \cdot \int \eta \beta^{\prime} \mathrm{e}^{-\beta}\left|\phi_{*}\right|^{2} .
\end{aligned}
$$

Also,

$$
\begin{aligned}
\int \mathrm{e}^{-\beta}\left|\phi_{*}^{\prime}\right|^{2} & =-\int \mathrm{e}^{-\beta} \phi_{*}\left(-\beta^{\prime} \phi_{*}^{\prime}+\phi_{*}^{\prime \prime}\right) \\
& =-\int \mathrm{e}^{-\beta} \phi_{*}\left(-\beta^{\prime} \phi_{*}^{\prime}+\beta^{\prime} \phi_{*}^{\prime}+\alpha_{\bullet}^{2} \phi_{*}-\Lambda_{*} \alpha_{\bullet} \beta^{\prime} \phi_{*}\right) \\
& =-\int \mathrm{e}^{-\beta} \phi_{*}^{2}\left(\alpha_{\bullet}^{2}-\Lambda_{*} \alpha_{\bullet} \beta^{\prime}\right) .
\end{aligned}
$$

Substituting (4.11) and (4.12) into (4.10), and simplifying, we get

$$
\begin{aligned}
I_{1} \cdot \underbrace{\int \mathrm{e}^{-\beta(\eta)}\left|\phi_{*}(\eta)\right|^{2}}_{\text {positive integral }}=\int \eta \mathrm{e}^{-\beta}\left|\phi_{*}^{\prime}\right|^{2} \cdot \int \mathrm{e}^{-\beta}\left|\phi_{*}\right|^{2}-\int \mathrm{e}^{-\beta}\left|\phi_{*}^{\prime}\right|^{2} \cdot \int \eta \mathrm{e}^{-\beta}\left|\phi_{*}\right|^{2} \\
=(\underbrace{\left(-\frac{1}{2} \int \beta^{\prime} \mathrm{e}^{-\beta}\left|\phi_{*}\right|^{2}\right)}_{\text {negative as } \beta^{\prime}>0}+\underbrace{\left(-\frac{1}{2} \mathrm{e}^{-\beta(\delta)} \phi_{*}^{2}(\delta)\right)}_{\text {negative constant }}) \int \mathrm{e}^{-\beta}\left|\phi_{*}\right|^{2} \\
+\underbrace{\left(\Lambda_{*} \alpha \bullet\right)}_{+ \text {ve constant }} \times \underbrace{\left(\int \eta \beta^{\prime} \mathrm{e}^{-\beta}\left|\phi_{*}\right|^{2} \cdot \int \mathrm{e}^{-\beta}\left|\phi_{*}\right|^{2}-\int \beta^{\prime} \mathrm{e}^{-\beta}\left|\phi_{*}\right|^{2} \cdot \int \eta \mathrm{e}^{-\beta}\left|\phi_{*}\right|^{2}\right)}_{\text {nonpositive by Lemma } 4.6 \text { with } g=\beta^{\prime}, f=\mathrm{e}^{-\beta}\left|\phi_{*}\right|^{2} \text { as } g^{\prime}=\beta^{\prime \prime} \leq 0} .
\end{aligned}
$$

Thus $I_{1}<0$. 
Let us now return to (4.9) and deal with the second term in the right-hand side. We have, with account of (2.3) and (2.7),

$$
\frac{\eta^{2} \gamma(\xi)^{2}}{1+\eta \gamma(\xi)} \leq\left\{\begin{array}{ll}
\eta^{2} \gamma(\xi)^{2} & \text { if } \gamma(\xi) \geq 0, \\
\frac{1}{1-A} \eta^{2} \gamma(\xi)^{2} & \text { if } \gamma(\xi)<0
\end{array} \quad \leq \max \left\{1, \frac{1}{1-A}\right\} \eta^{2} \gamma(\xi)^{2}\right.
$$

and so

$$
\alpha_{\bullet}^{2} \int_{-r}^{r} \int_{0}^{\delta} \frac{\eta^{2} \gamma(\xi)^{2}}{1+\eta \gamma(\xi)} \mathrm{e}^{-\beta(\eta)}\left|\phi_{*}(\eta)\right|^{2} \mathrm{~d} \eta \mathrm{d} \xi \leq I_{2} \int_{-r}^{r} \gamma(\xi)^{2} \mathrm{~d} \xi
$$

where

$$
I_{2}:=\max \left\{1, \frac{1}{1-A}\right\} \alpha_{\bullet}^{2} \int_{0}^{\delta} \eta^{2} \mathrm{e}^{-\beta(\eta)}\left|\phi_{*}(\eta)\right|^{2} \mathrm{~d} \eta \geq 0
$$

Thus, as $\gamma(\xi)$ vanishes for $|\xi|>R$, we have

$$
D_{\gamma}=I_{1} \int \gamma(\xi) \mathrm{d} \xi+I_{2} \int \gamma(\xi)^{2} \mathrm{~d} \xi=\left(-I_{1}\right)\left(C_{\beta} \int \gamma(\xi)^{2} \mathrm{~d} \xi-\int \gamma(\xi) \mathrm{d} \xi\right)
$$

where

$$
C_{\beta}=\frac{I_{2}}{-I_{1}}=\frac{\max \left\{1, \frac{1}{1-A}\right\} \alpha_{\bullet}^{2} \int_{0}^{\delta} \eta^{2} \mathrm{e}^{-\beta(\eta)}\left|\phi_{*}(\eta)\right|^{2} \mathrm{~d} \eta}{\int_{0}^{\delta} \eta \mathrm{e}^{-\beta(\eta)}\left(\left|\phi_{*}^{\prime}(\eta)\right|^{2}-\alpha_{\bullet}^{2}\left|\phi_{*}(\eta)\right|^{2}\right) \mathrm{d} \eta}
$$

is a positive constant.

As soon as (4.2) holds, $D_{\gamma}$ is negative, and so (4.5) holds. This proves Theorem 4.1 .

Finally, it is sufficient to note that (4.3) implies $\int \gamma(\xi)^{2} \mathrm{~d} \xi<2 R c_{\beta, R} \int \gamma(\xi) \mathrm{d} \xi$, which proves Corollary 4.2.

5. Conclusions. It has been shown that a trapped mode is possible in the model presented here. To increase confidence that such modes exist on real coasts further work is clearly required to demonstrate that this mode is not an artifact of the modelling assumptions. However these assumptions are the usual ones for the simple theory of CSWs and extensions to include stratification and more realistic boundary conditions have not in general contradicted them [LBMy]. The result here suggests that it would be of interest to compare low-frequency velocity records in the neighborhood of capes with those on nearby straight coasts to determine whether there is indeed enhanced energy at the cape. Both the above endeavors are being pursued.

\section{REFERENCES}

[AsPaVa] A. Aslanyan, L. Parnovski, and D. Vassiliev, Complex resonances in acoustic waveguides, Quart. J. Mech. Appl. Math., 53 (2000), pp. 429-447.

[Da] E. B. Davies, Spectral Theory and Differential Operators, Cambridge University Press, Cambridge, UK, 1995.

[DaPa $]$ E. B. Davies And L. PARnovski, Trapped modes in acoustic waveguides, Quart. J. Mech. Appl. Math., 51 (1998), pp. 477-492. 
$[\mathrm{DiKr}]$

[DuEx]

[EvLeVa]

[ExSe]

$[\mathrm{Ha}]$

$[\mathrm{Jo1}]$

[Jo2]

$[\mathrm{KrKr}]$

$[\mathrm{KrTA}]$

$[\mathrm{Pe}]$

$[\mathrm{StHu} 2]$

[StHuSaTrZa]

[StJo1]

[StJo2]
J. DitTrich And J. KŘÍž, Curved planar quantum wires with Dirichlet and Neumann boundary conditions, J. Phys. A, 35 (2002), pp. L269-L275.

P. DuClos AND P. EXNER, Curvature-induced bound states in quantum waveguides in two and three dimensions, Rev. Math. Phys., 7 (1995), pp. 73-102.

D. V. Evans, M. Levitin, And D. VAssiliev, Existence theorems for trapped modes, J. Fluid Mech., 261 (1994), pp. 21-31.

P. Exner And P. ŠEBA, Bound states in curved quantum waveguides, J. Math. Phys., 30 (1989), pp. 2574-2580.

B. V. HAMON, Continental shelf waves and the effect of atmospheric pressure and wind stress on sea level, J. Geophys. Res., 71 (1966), pp. 2883-2893.

E. R. Johnson, A conformal-mapping technique for topographic-wave problemssemi-infinite channels and elongated basins, J. Fluid Mech., 177 (1987), pp. 395-405.

E. R. Johnson, Topographic waves in open domains. I. Boundary conditions and frequency estimates, J. Fluid Mech., 200 (1989), pp. 69-76.

E. R. Johnson, Low-frequency barotropic scattering on a shelf bordering an ocean, J. Phys. Oceanogr., 21 (1991), pp. 720-727.

D. KReJČIǏíK AND J. KŘÍž, On the spectrum of curved planar waveguides, Publ. Res. Inst. Math. Sci., 41 (2005), pp. 757-791.

D. KrejČIŘík And R. Tiedra DE AldecoA, The nature of the essential spectrum in curved quantum waveguides, J. Phys. A, 37 (2004), pp. 5449-5466.

H. Lamb, Hydrodynamics, 6th ed., Cambridge University Press, Cambridge, UK, 1932.

P. H. LeBlond and L. A. Mysak, Waves in the Ocean, Elsevier, Amsterdam, 1978.

J. Pedlosky, Geophysical Fluid Dynamics, Springer, Berlin, 1986.

T. F. Stocker AND K. Hutter, One-dimensional models for topographic Rossby waves in elongated basins on the f-plane, J. Fluid Mech., 170 (1986), pp. $435-459$.

T. F. Stocker And K. HutTeR, Topographic waves in rectangular basins, J. Fluid Mech., 185 (1987), pp. 107-120.

K. Stocker, K. Hutter, G. Salvade, J. Trösch, and F. Zamboni, Observations and analysis of internal seiches in the southern basin of Lake of Lugano, Ann. Geophys. B, 5 (1987), pp. 553-568.

T. F. Stocker And E. R. Johnson, Topographic waves in open domains. II. Bay modes and resonances, J. Fluid Mech., 200 (1989), pp. 77-93.

T. F. Stocker And E. R. Johnson, The trapping and scattering of topographic waves by estuaries and headlands, J. Fluid Mech., 222 (1991), pp. 501-524.

J. Trösch, Finite element calculation of topographic waves in lakes, in Proceedings of the 4th International Conference on Applied Numerical Modeling (Tainan, Taiwan, 1984), Science and Technology Series 63, H. M. Hsia, Y. L. Chou, S. Y. Wang, and S. J. Hsieh, eds., American Astronautical Society, 1986, pp. 307-311. 